\title{
Super-short fission mode in fermium isotopes
}

\author{
M. Albertsson, ${ }^{1}$ B. G. Carlsson, ${ }^{1}$ T. Døssing, ${ }^{2}$ P. Möller, ${ }^{1,3}$ J. Randrup,${ }^{4}$ and S. Åberg $\oplus^{1, *}$ \\ ${ }^{1}$ Mathematical Physics, Lund University, S-221 00 Lund, Sweden \\ ${ }^{2}$ Niels Bohr Institute, University of Copenhagen, DK-2100 Copenhagen $\emptyset$, Denmark \\ ${ }^{3}$ P. Moller Scientific Computing and Graphics, P.O. Box 75009, Honolulu, Hawaii 96836, USA \\ ${ }^{4}$ Nuclear Science Division, Lawrence Berkeley National Laboratory, Berkeley, California 94720, USA
}

(Received 15 May 2021; accepted 9 December 2021; published 27 December 2021)

\begin{abstract}
The so-called super-short fission mode, in which a nucleus divides nearly symmetrically into two unusually energetic fragments, competes favorably with the standard asymmetric fission mode for spontaneous fission of a limited number of nuclei near ${ }^{264} \mathrm{Fm}$ but it quickly fades away at finite excitations. We investigate the energy-dependent competition between those two fission modes for even fermium isotopes from ${ }^{254} \mathrm{Fm}$ to ${ }^{268} \mathrm{Fm}$, using the Metropolis method to simulate the strongly damped fission dynamics being driven by shape- and energy-dependent level densities. The origin of the super-short mode is discussed and its effects on the fragment mass distribution, the total fragment kinetic energy, and the neutron multiplicity are calculated. Generally good agreement with the available data is obtained.
\end{abstract}

DOI: 10.1103/PhysRevC.104.064616

\section{INTRODUCTION}

Nuclear fission presents a rich variety of challenging theoretical problems in which structure and dynamics are interwoven [1]. A particularly intricate problem has long been posed by the appearance of the so-called super-short fission mode in certain fermium isotopes. In this paper, we map out the domain of this fragile mode with respect to the mass number and the excitation energy of the fissioning nucleus.

The lighter fermium isotopes fission by a "standard mode" in which the mass distribution of the fragments is asymmetric and the total fragment kinetic energy (TKE) is fairly low; the associated scission configurations are rather elongated. However, in the neighborhood of ${ }^{264} \mathrm{Fm}$ the nucleus may also divide nearly symmetrically into fragments that lie close to the doubly magic nucleus ${ }^{132} \mathrm{Sn}$. The corresponding scission configurations then consist of two approximately spherical protofragments and they are thus unusually compact (hence the name "super-short" for this mode [2], sometimes called "compact" mode; see, e.g., Ref. [3]); as a result, the fragments experience a stronger Coulomb repulsion and hence acquire higher kinetic energies.

The occurrence of bimodal fission in the fermium region was first noted experimentally 50 years ago $[4,5]$ and the phenomenon has since been explored more thoroughly [6-11].

*Corresponding author: sven.aberg@matfys.lth.se

Published by the American Physical Society under the terms of the Creative Commons Attribution 4.0 International license. Further distribution of this work must maintain attribution to the author(s) and the published article's title, journal citation, and DOI. Funded by Bibsam.
The super-short mode grows more prominent as ${ }^{264} \mathrm{Fm}$ is approached and it is expected that it again subsides for still heavier isotopes. Furthermore, for the nuclei in which the mode appears, it quickly loses importance with increasing excitation energy. Thus, the super-short mode is rather delicate and the present work seeks to determine its quantitative importance in fermium isotopes as a function of mass number and excitation energy.

The bimodal fission phenomenon has been studied with a variety of theoretical approaches, most notably static mean-field models [3,12-18], models based on density functional theory $[19,20]$, the time-dependent generatorcoordinate method [21], and Langevin transport treatments [22-25]. Generally, these calculations reproduce the presence of the two fission modes (standard and super-short), but the dependence on excitation energy has not been fully explored. For example, the generator coordinate calculations are carried out at zero temperature, while the Langevin treatments have been restricted to above-barrier fission.

In this study, we employ methods developed earlier to study the presence (and cause) of the super-short fission mode in even fermium isotopes and how it evolves with excitation energy and neutron number. In addition to calculating the fragment mass distributions, we extract the associated TKE distributions and calculate the number of neutrons evaporated from the excited fragments, observables that exhibit characteristic mutual correlations.

The model is briefly described in Sec. II, the results are presented in Sec. III, and in Sec. IV we summarize our study.

\section{MODEL}

The fission process is described as a dissipative evolution of the nuclear shape [26], represented by the 5 degrees of 
freedom of the three-quadratic-surface parametrization [27]: the overall elongation as measured by the dimensionless quadrupole moment, $q_{2}$; the degree of reflection asymmetry, $\alpha$; the radius of the neck between the two nascent fragments, $c$; and the deformations of the two spheroidal endcaps of those, $\varepsilon_{L}$ and $\varepsilon_{H}$. The dimensionless elongation parameter $q_{2}$ is defined from the quadrupole moment of a homogeneous, sharp-surfaced volume, $Q_{2}$, as $q_{2}=\left(4 \pi Q_{2}\right) /\left(3 Z R_{A}^{2}\right)$ with $R_{A}=1.2 A^{1 / 3} \mathrm{fm}$. The grid was set up as described in Ref. [28] with an extension to larger quadrupole shapes including more than 6 million grid points, as compared to the about 5 million points used in Ref. [28]. The $q_{2}$ coordinate thus extends from $q_{2}=0$ to 20 divided into 52 intervals (as was also used in Refs. [29,30]). For each combination of $q_{2}$, $\alpha, \varepsilon_{L}$, and $\varepsilon_{H} 15$ neck radii, $c$, are considered.

The shape evolution is simulated by the Metropolis method [31] in which the system executes a random walk on an effective five-dimensional (5D) potential-energy surface consisting of a macroscopic energy plus microscopic pairing- and shell-correction energies that are gradually suppressed with increasing total energy [32].

The macroscopic-microscopic approach using the finiterange liquid drop model (FRLDM) is used to generate the energies and spectra for the shapes in the 5D energy surface. The pairing gap in this model is surface-area dependent, which gives a smooth transition of the pairing-correction energy as the nucleus divides into two fragments [33].

For each case considered, $10^{5}$ fission events are generated by following the Metropolis walks from the initial shape until a scission configuration is reached. As scission is approached, the fragment mass numbers $A_{L}$ and $A_{H}$ as determined by the asymmetry parameter $\alpha$, are frozen in when the neck radius has shrunk to $c_{0}=2.5 \mathrm{fm}[31,32]$; only fragments with even numbers of protons and neutrons are considered here, with the nucleon numbers being determined by approximately matching the $Z / N$ ratio of the fissioning nucleus. Scission is reached at $c_{\mathrm{sc}}=1.5 \mathrm{fm}[29,30]$ and the system is then replaced by two protofragments whose spheroidal shapes are given by the values of $\varepsilon_{L}$ and $\varepsilon_{H}$ at scission. The available energy is partitioned microcanonically between the fragments based on shape-dependent microscopic level densities [29].

As the protofragments separate and accelerate, their initially distorted shapes relax to their equilibrium forms and the intrinsic fragment energies are increased correspondingly. For a given mass partition, $A_{0} \rightarrow A_{L}+A_{H}$, at the excitation energy $E_{0}^{*}$ the $Q$ value, $Q_{\mathrm{LH}}^{*}=M_{0}+E_{0}^{*}-M_{L}-M_{H}$, represents the energy available for the total fragment kinetic energy, TKE, and the combined excitations of the two primary fragments, $\mathrm{TXE}=E_{L}^{\mathrm{intr}}+E_{H}^{\mathrm{intr}}, Q_{\mathrm{LH}}^{*}=\mathrm{TKE}+\mathrm{TXE}$. The fragment excitation energies are eventually disposed of by neutron evaporation and $\gamma$ de-excitation [29].

The Metropolis random walk method provides a simulation of overdamped motion. Once an initial shape has been chosen, each walk proceeds on its own, governed by the local level densities and by the Metropolis algorithm, until scission is reached. The distributions of physical observables are subsequently calculated by sampling many walks. Most notably, the mass distribution of fissioning nuclei has been calculated over wide intervals of neutron and proton number $[32,34,35]$. In

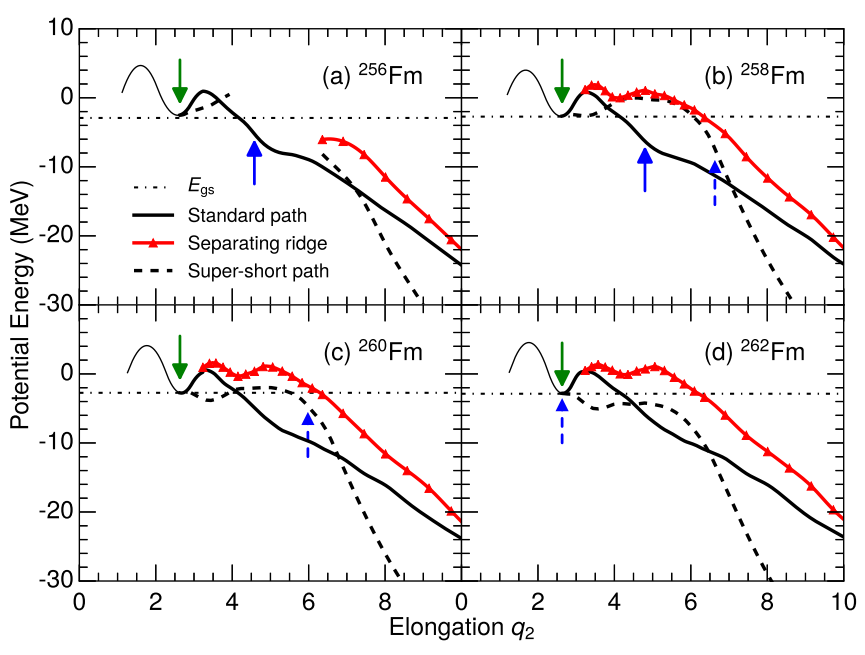

FIG. 1. The potential energy along the paths for the standard (solid) and the super-short (dashed) fission modes, as function of the overall quadrupole moment $q_{2}$ for ${ }^{256} \mathrm{Fm}$ (a), ${ }^{258} \mathrm{Fm}$ (b), ${ }^{260} \mathrm{Fm}$ (c), and ${ }^{262} \mathrm{Fm}(\mathrm{d})$; the two paths are separated by a ridge (red line). The ground-state energy is shown by the horizontal line. The shape evolutions start at the isomeric minima (down-pointing arrows). For spontaneous fission, the extra energy $\Delta E$ is reset typically at the up-pointing arrows.

order to interpret the results obtained, it is valuable to identify the most probable fission paths, running along valley bottoms and diffusing over intermediate ridges or saddle points. This was illustrated in Ref. [36] for the fission of ${ }^{236} \mathrm{U}$. The fission paths are found by identifying the local minima for each $q_{2}$ value in the energy surface and smoothly following these minima as $q_{2}$ increases. The ridges separating the local minima are found using the immersion method [28].

For the fermium isotopes under study here, two qualitatively different paths are apparent: "standard" paths leading to very elongated mass-asymmetric scission configurations and paths leading to nearly mass-symmetric scission configurations that are very compact. For ${ }^{256-262} \mathrm{Fm}$, Fig. 1 depicts the potential energy along those two fission paths, together with the ridge separating them, as functions of the elongation $q_{2}$. The corresponding curves for ${ }^{254} \mathrm{Fm}$ (not shown) are similar to those of ${ }^{256} \mathrm{Fm}$. Those for ${ }^{264,266,268} \mathrm{Fm}$ (not shown) are all similar to those for ${ }^{262} \mathrm{Fm}$. For each isotope, its ground-state energy is marked by the horizontal line and its first barrier peaks at $q_{2} \approx 1.4$. (Similar results have been obtained on the basis of the constrained Hartree-Fock model $[15,19,20]$.)

For the curves shown in Fig. 1, the angular momentum is set equal to $I=0$, as is appropriate for spontaneous fission of even-even nuclei. For neutron-induced fission at the low energies considered here, angular momenta values from 0 up to about $6 \hbar$ will be carried by the nucleus prior to fission. Considering the large moments of inertia of these very heavy nuclei, the energy associated with the angular momentum will be typically less than $100 \mathrm{keV}$, which is much smaller than the typical shell- and pairing-correcion energies. A careful inclusion of the angular momentum of the nucleus prior to fission would then have practically no effect on the present result, as also shown for the fission of ${ }^{236} \mathrm{U}$ in Ref. [36]. 
As is characteristic for the actinides, the fermium isotopes have isomeric minima (at $q_{2} \approx 2.5$ ); these lie only slightly above of the respective ground-state minima. Generally, the nuclear shapes will evolve through the neighborhood of the isomeric minimum so, as we are not concerned with the fission half-lives, the evolutions may be started from there. Only the standard fission path seamlessly connects scission configurations to the isomeric minimum in ${ }^{254,256} \mathrm{Fm}$, while for ${ }^{258-268} \mathrm{Fm}$ both paths are connected to the isomeric minimum. (A more asymmetric path competes in energy with the standard path but is ignored because it plays only a minor role in the dynamics.) Scission occurs at $q_{2} \approx 13$ for the standard path but already at $q_{2} \approx 8$ for the super-short path. The random walks fully utilize all five shape degrees of freedom and may switch from one fission path to another if energetically allowed.

A special challenge is presented by the presence of classically forbidden regions through which the shape must tunnel at the lowest energies considered. For this problem, we employ a modified Metropolis walk ( $\Delta E$ method) in which the total energy is initially increased by an amount $\Delta E$ sufficient to overcome the lowest barrier and we then reduce it back to its physical value once it becomes possible. In this way, the diffusive paths divide into two different shape ensembles, one for each fission mode, whose further evolution can then be continued until scission by use of the standard Metropolis method.

As a check, we alternatively estimate the relative probability for entering each of the two valleys by means of a simple WKB treatment of spontaneous fission for each of the isotopes considered. After the tunneling has been completed, the evolution is continued by the standard Metropolis method starting from the shape ensemble obtained by the $\Delta E$ method at the respective exit values of $q_{2}$.

A detailed investigation of tunneling under a fivedimensional barrier would be too involved for the present study, so we estimate the branching ratio by evaluating the WKB action integrals along the minimum paths through the potential landscape (see Fig. 1), taking the corresponding mass parameters from Ref. [14].

The two different methods for estimating the branching ratio for spontaneous fission (sf) yield very similar results, except for ${ }^{258} \mathrm{Fm}$. For that nucleus, WKB favors the supershort mode because the inertial mass is relatively small along the path to the super-short valley, resulting in a branching of $\approx 75 \%$ to the super-short valley and $\approx 25 \%$ to the asymmetric standard valley. However, the $\Delta E$ method cannot reproduce this feature because it is sensitive only to the potential and so it favors the standard mode by $\approx 93 \%$.

For the two lightest isotopes considered, ${ }^{254,256} \mathrm{Fm}$, both the WKB calculation and the $\Delta E$ method lead exclusively to the standard asymmetric valley for sf [a typical starting position in this valley is marked by the blue arrow in Fig. 1(a)].

For ${ }^{258,260} \mathrm{Fm}$ the paths to the two valleys can be identified already from the isomeric minimum. As discussed above, we expect the super-short mode to be favored for ${ }^{258} \mathrm{Fm}(\mathrm{sf})$. For ${ }^{260} \mathrm{Fm}$ the super-short is even more favored because the barrier between the isomeric minimum and the super-short

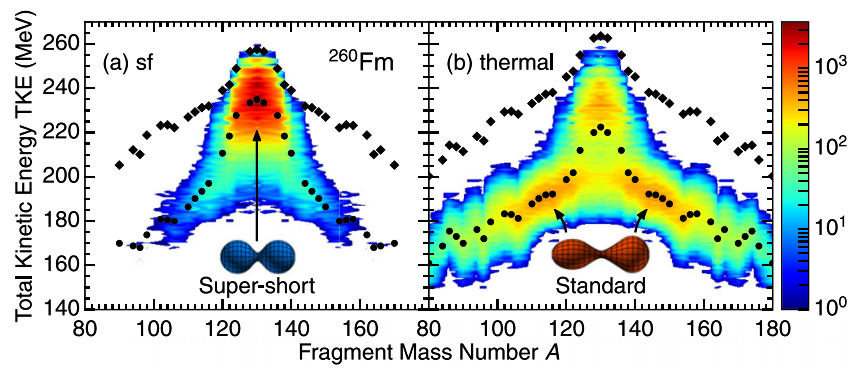

FIG. 2. Contour plots (on a logarithmic scale) of the number of fission events for ${ }^{260} \mathrm{Fm}$ in the A-TKE plane based on $10^{5}$ spontaneous or thermal fission events. Also shown are $Q_{\mathrm{LH}}^{*}$ (filled diamonds) and $\overline{\operatorname{TKE}}(A)$ (filled circles). Typical scission shapes are shown for the compact, symmetric super-short and the elongated, asymmetric standard modes.

valley is very low, less than $1 \mathrm{MeV}$, and the branching ratio is practically $100 \%$.

\section{RESULTS}

We have performed Metropolis random-walk calculations for the even fermium isotopes ${ }^{254-268} \mathrm{Fm}$ at various excitation energies with the above described starting conditions and studied a variety of fission observables. In particular, we have extracted the fragment mass distribution, the TKE distribution, and $\bar{v}$, the average total number of neutrons evaporated from the fragments.

Figure 2 shows the joint distribution of fragment mass number $A$ and total kinetic energy TKE for both spontaneous [Fig. 2(a)] and thermal [Fig. 2(b)] fission of ${ }^{260} \mathrm{Fm}$, $\left[{ }^{259} \operatorname{Fm}\left(n_{\text {th }}, f\right]\right)$. At the lower energy [Fig. 2(a)], most events are approximately mass symmetric and have large TKE. These arise primarily from the super-short fission mode, in which the scission configurations are compact, consisting of two nearly spherical tinlike fragments that acquire high kinetic energies. The increase of the energy by $6.13 \mathrm{MeV}$ [Fig. 2(b)] enables the system to access also the standard fission mode, resulting in a very different joint $A$-TKE distribution, with many asymmetric events that have significantly lower TKE.

Consequently, the overall TKE distribution for ${ }^{259} \mathrm{Fm}\left(n_{\text {th }}, f\right)$ [which can be obtained by projecting the joint distribution shown in Fig. 2(b)] has two distinct components, one centered at TKE $\approx 190 \mathrm{MeV}$ and another centered at $\mathrm{TKE} \approx 228 \mathrm{MeV}$. Furthermore, the distribution of the $q_{2}$ values of the scission configurations is also bimodal, being well described by two separated Gaussians having their peaks at $q_{2} \approx 13$ and at $q_{2} \approx 8$. The events in the first component $\left(q_{2} \approx 13\right)$ belong to the standard fission mode, whose elongated scission configurations (which typically consist of a prolate light fragment and a slightly oblate heavy fragment) lead to rather low TKE values, while the other component $\left(q_{2} \approx 8\right)$ represents the super-short events which have very compact scission configurations (consisting of two nearly equal and approximately spherical protofragments) that lead to high TKE. Typical scission shapes for the two modes are shown in Fig. 2. 


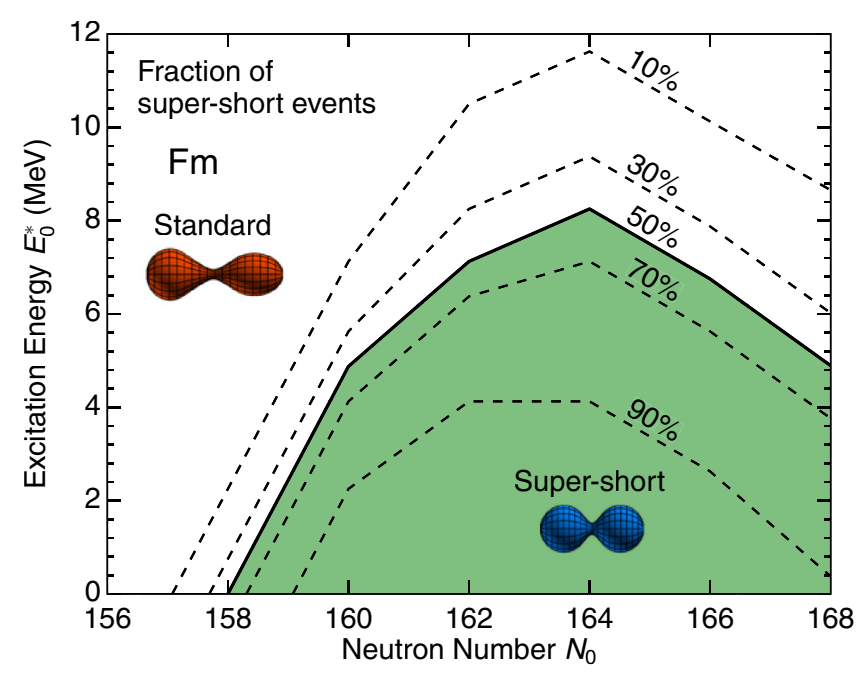

FIG. 3. Contour plot $f^{\text {ss }}$, the calculated fraction of fissions via the super-short mode, in fermium isotopes in the $N_{0}-E_{0}^{*}$ plane. The solid line marks the $50 \%$ contour where the super-short mode and the standard mode contribute equally. Typical scission shapes for the two modes are shown.

In the dynamical calculations, the two different fission modes can be separated on the basis of the elongation of the scission configuration, $q_{2}^{\mathrm{sc}}$, and the fraction of super-short events, $f^{\text {ss }}$, can thus be obtained. For the cases shown in Fig. 2, $f_{\mathrm{sf}}^{\mathrm{ss}} \approx 95 \%$ and $f_{\mathrm{th}}^{\mathrm{ss}} \approx 20 \%$.

In order to ascertain the role played by the super-short mode in fermium, we have extracted $f^{\text {ss }}$ for a number of isotopes with an even neutron number $N_{0}$ and a range of initial excitation energies $E_{0}^{*}$. The result is summarized in Fig. 3 in the form of a contour plot of the super-short fraction $f^{\mathrm{ss}}\left(N_{0}, E_{0}^{*}\right)$.

Figure 3 shows that the super-short mode dominates for spontaneous fission of ${ }^{258-268} \mathrm{Fm}$, but the mode is fragile and for ${ }^{258} \mathrm{Fm}$ it quickly subsides with increasing energy. As $N_{0}$ approaches 164 , the mode grows increasingly more resilient, up to ${ }^{264} \mathrm{Fm}$, for which it remains dominant until $E_{0}^{*} \approx 8$ $\mathrm{MeV}$. That the super-short mode should be most significant for that isotope is to be expected because symmetric fission leads to two doubly magic ${ }^{132} \mathrm{Sn}$ fragments. For still heavier isotopes, the mode gradually loses prominence and appears to be insignificant beyond ${ }^{272} \mathrm{Fm}$.

The calculated variation in the resilience of the super-short fission mode with increasing energy can be understood from Fig. 1. At low energies, the walks are confined within the super-short valley but with increasing energy it becomes ever easier to cross over the ridge into the asymmetric standard valley, as we discuss in more detail below. Such leakage from the super-short to the standard valley on the way toward scission reduces $f^{\text {ss }}$ relative to its value just above the barrier.

The potential energy along the super-short fission path slopes downward relatively gently beyond $q_{2} \approx 5$, causing the shape evolution to have a predominantly diffusive character. This offers favorable opportunities for the shape to cross over into the valley associated with the standard mode and once such a transition has been made, the likelihood of returning to the super-short valley is very small, because the slope along the standard fission path is significantly steeper, thus providing a strong driving force toward scission.

Because the energy difference between the super-short valley and the ridge line is small throughout much of the diffusive region, even a small amount of excitation energy may induce transitions to the standard valley. For example, for ${ }^{260} \mathrm{Fm}$ (Fig. 3) the super-short fraction $f^{\text {ss }}$ is shrinking rapidly as the energy is increased, dropping from $95 \%$ at $E_{0}^{*}=0$ to $20 \%$ at $6 \mathrm{MeV}$.

The potential energy along the super-short fission path (in the interval $q_{2}=4-6$ ) decreases with increasing neutron number $N_{0}$ from 158 to 162 [see Figs. 1(b)-1(d)] and reaches its lowest values for 164. The ridge energy is independent of $N_{0}$, so transitions from the super-short to the standard path require higher energies with increasing neutron number, with a maximum at 164, thus making the super-short mode in those isotopes correspondingly more prominent.

If the excitation energy is increased substantially, the microscopic effects erode and fission proceeds in a symmetric liquid-drop-like fashion. Shell structure in the fragment nuclei is expected to disappear at temperatures above $1.5 \mathrm{MeV}$ [37], suggesting this change may appear above $E_{0}^{*} \approx 40$ $\mathrm{MeV}$. This estimate was confirmed in the full Metropolis calculation.

The presence of the super-short fission mode has a correlated effect on several observables, particularly the fragment mass distribution, the TKE, and the neutron multiplicity. As noted above, the two fission modes lead to very different mass distributions, the standard mode yielding asymmetric mass distributions (peaked at $A_{L}=108$ and $A_{H}=146$ for ${ }^{254} \mathrm{Fm}$ ), while the super-short mode yields narrow symmetric distributions. Figure 4 shows the primary fragment mass distribution resulting from ${ }^{254-260} \mathrm{Fm}(\mathrm{sf})$ and ${ }^{254-260} \mathrm{Fm}\left(n_{\text {th }}\right)$. The yields for spontaneous fission (left panels) exhibit a sudden change as $N_{0}$ is increased from 156 to 158 . For ${ }^{254,256} \mathrm{Fm}$, the standard mode completely dominates, resulting in asymmetric yield distributions, while the super-short dominates for ${ }^{258} \mathrm{Fm}$ and ${ }^{260} \mathrm{Fm}$ (with $55 \%$ and $95 \%$, respectively), yielding narrow symmetric fragment mass distributions.

At $E_{0}^{*} \approx 6 \mathrm{MeV}$, corresponding to thermal fission, the transition from the standard to the super-short fission mode occurs at a larger $N_{0}$, as is shown in Fig. 3. Compared to the results for spontaneous fission of ${ }^{258,260} \mathrm{Fm}$, the mass distribution is drastically wider at this energy, at which also the standard mode contributes to the symmetric mass yield.

While the experimental data appear to support the general characteristic features of the calculated results, a few differences may be noted: (1) the peaks of the asymmetric mass splits for ${ }^{254-256} \mathrm{Fm}$ are wider in the calculation than in the data [Figs. 4(a), 4(d), and 4(e)]; and (2) the measured yields for ${ }^{257} \mathrm{Fm}\left(n_{\mathrm{th}}, f\right)$ have more events at symmetry than calculated.

The sharp transition from the standard to the super-short mode with increasing $N_{0}$ also causes a correspondingly rapid change in the TKE distribution. This is illustrated for spontaneous fission in the left panels of Fig. 5: a single low-TKE peak for ${ }^{254,256} \mathrm{Fm}$ (sf) changes via a bimodal distribution for ${ }^{258} \mathrm{Fm}$ (sf) to a single high-TKE peak for ${ }^{260} \mathrm{Fm}$ (sf). 


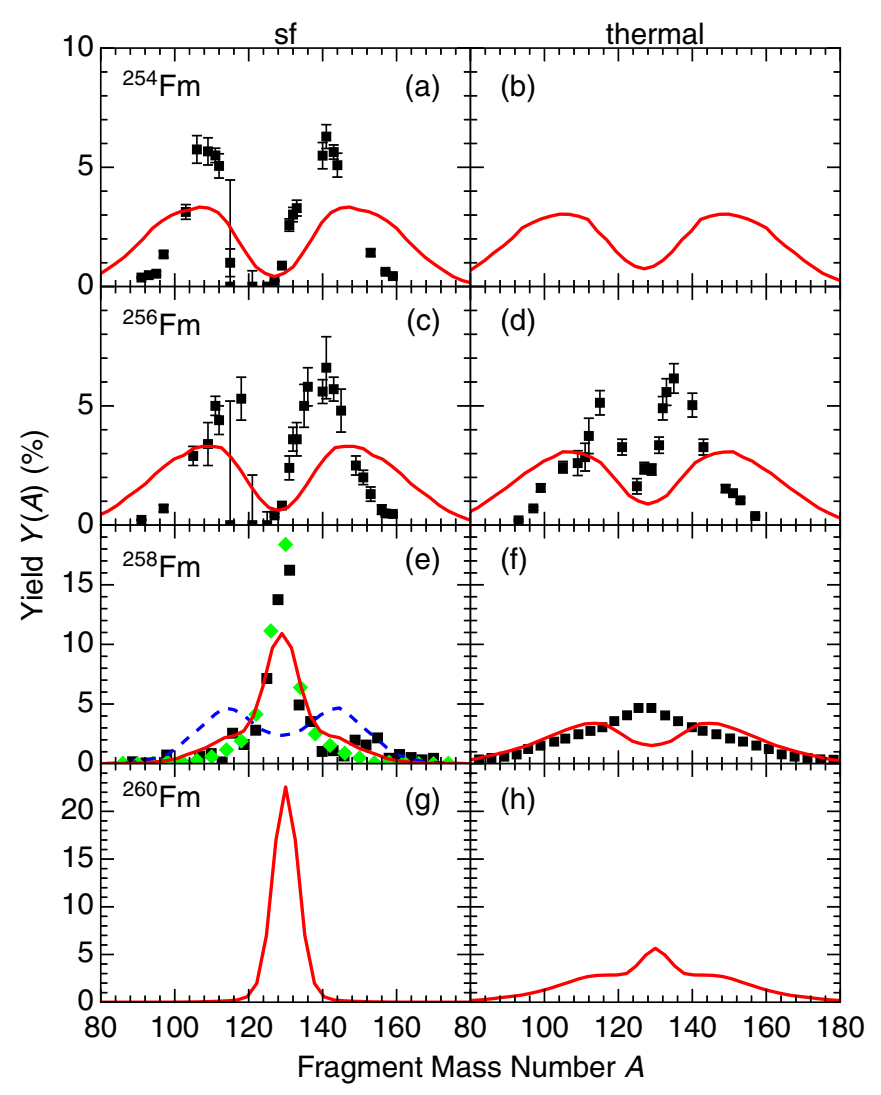

FIG. 4. Primary fragment mass yields for spontaneous and thermal fission for ${ }^{254} \mathrm{Fm}\left[(\mathrm{a})\right.$, (b)], ${ }^{256} \mathrm{Fm}[(\mathrm{c}),(\mathrm{d})],{ }^{258} \mathrm{Fm}[(\mathrm{e}),(\mathrm{f})]$, and ${ }^{260} \mathrm{Fm}[(\mathrm{g}),(\mathrm{f})]$. The calculated results are shown by solid red curves; for ${ }^{258} \mathrm{Fm}$ the sf result from the $\Delta E$ method is shown in panel (e) as a blue dashed line. The experimental data for sf are shown in panel (a) for ${ }^{254} \mathrm{Fm}$ [38], in panel (c) for ${ }^{256} \mathrm{Fm}$ [39], and in panel (e) for ${ }^{258} \mathrm{Fm}$ [40] as black squares; data from Ref. [11] are shown in panel (e) as green diamonds. Thermal fission data are shown as black squares in panel (d) for ${ }^{256} \mathrm{Fm}\left[{ }^{255} \mathrm{Fm}\left(n_{\text {th }}, f\right)\right][41]$ and in panel (f) for ${ }^{258} \mathrm{Fm}\left[{ }^{257} \mathrm{Fm}\left(n_{\mathrm{th}}, f\right)\right]$ [5]. The mass yields are for the product nuclei.

For ${ }^{258} \mathrm{Fm}(\mathrm{sf})$, the super-short fraction is $f^{\text {ss }}=55 \%$ and the two TKE peaks are located at 188 and $236 \mathrm{MeV}$ when the WKB method is employed. The $\Delta E$ method also yields a bimodal distribution but favors the standard mode somewhat and leads to a wider fragment mass distribution and lower TKE values [see Figs. 4(e) and 5(e)].

At $E_{0}^{*} \approx 6 \mathrm{MeV}$ (thermal fission) the standard mode completely dominates for ${ }^{254,256,258} \mathrm{Fm}$ and yields a single low-TKE peak at $188 \mathrm{MeV}$ [Figs. 5(b), 5(d), and 5(f)]. For ${ }^{260} \mathrm{Fm}$, the super-short fraction is $f^{\mathrm{ss}}=20 \%$ creating a smaller TKE peak at $230 \mathrm{MeV}$ in addition to the larger peak at $188 \mathrm{MeV}$.

For the studied eight fermium isotopes, the overall average TKE values, $\overline{\mathrm{TKE}}$, are shown in Fig. 6(a) for three excitation energies, $E_{0}^{*}=0(\mathrm{sf}), E_{0}^{*} \approx 6 \mathrm{MeV}$ (thermal), and $E_{0}^{*}=$ $15 \mathrm{MeV}$ (with second-chance fission ignored). The transition from the standard mode to the super-short mode appears as a rapid increase in $\overline{\mathrm{TKE}}$. This trend also appears in the data on ${ }^{259,260}$ Md (sf).

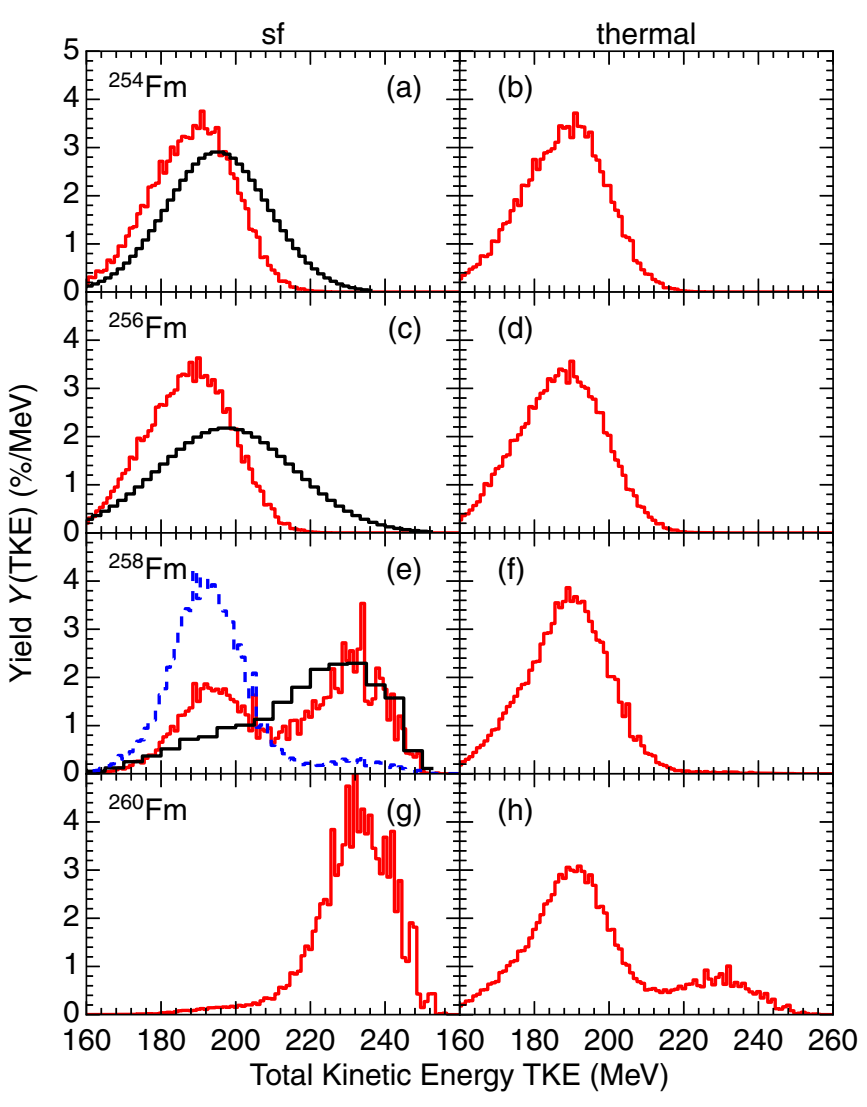

FIG. 5. TKE distributions for the same cases shown in Fig. 4. Calculated results are shown by solid red curves; for ${ }^{258} \mathrm{Fm}$ the sf result from the $\Delta E$ method is shown in panel (e) by a blue dashed line. Data for sf are shown by solid black curves for ${ }^{254} \mathrm{Fm}$ (a) [38], ${ }^{256} \mathrm{Fm}$ (b) [42], and ${ }^{258} \mathrm{Fm}$ (e) [11].

The result is readily understood from Fig. 3, from which the super-short fraction $f^{\text {ss }}$ dependence on $N_{0}$ can be inferred at the three energies. A sudden increase of $f^{\text {ss }}$ causes a corresponding increase in $\overline{\mathrm{TKE}}$. This occurs at $N_{0}=158$ for spontaneous fission and at $N_{0}=162$ for thermal fission. At the latter energy, the drop in $f^{\text {ss }}$ at $N_{0}=166$ appears as a drop in the $\overline{\mathrm{TKE}}$. For the highest energy, all isotopes are found to fission in the standard mode and TKE remains low.

Decay from the excited fully accelerated fragments carries information about the excitation energy of the fragments. In the present calculations, the microcanonical excitation of each fragment at scission is accounted for by the state density, obtained by summing the level density over all angular momenta $I$, multiplied by the factor $(2 I+1)$. The typical magnitude of the angular momentum in each fragment is about 6-8 $\hbar$, generated by the thermal excitation at scission. Such values are qualitatively in agreement with $\gamma$-ray multiplicity data $[53,54]$. In principle, the angular momenta of the two fragments and their relative orbital angular momenta should be coupled as three quantal angular momentum vectors to yield the small total angular momentum vector of length $I=0$ to about $6 \hbar$ carried by the fissioning nucleus. This is not done in the present calculations. Instead, the average rotational energy of the order of 1-2 MeV in each fragment is subtracted from 


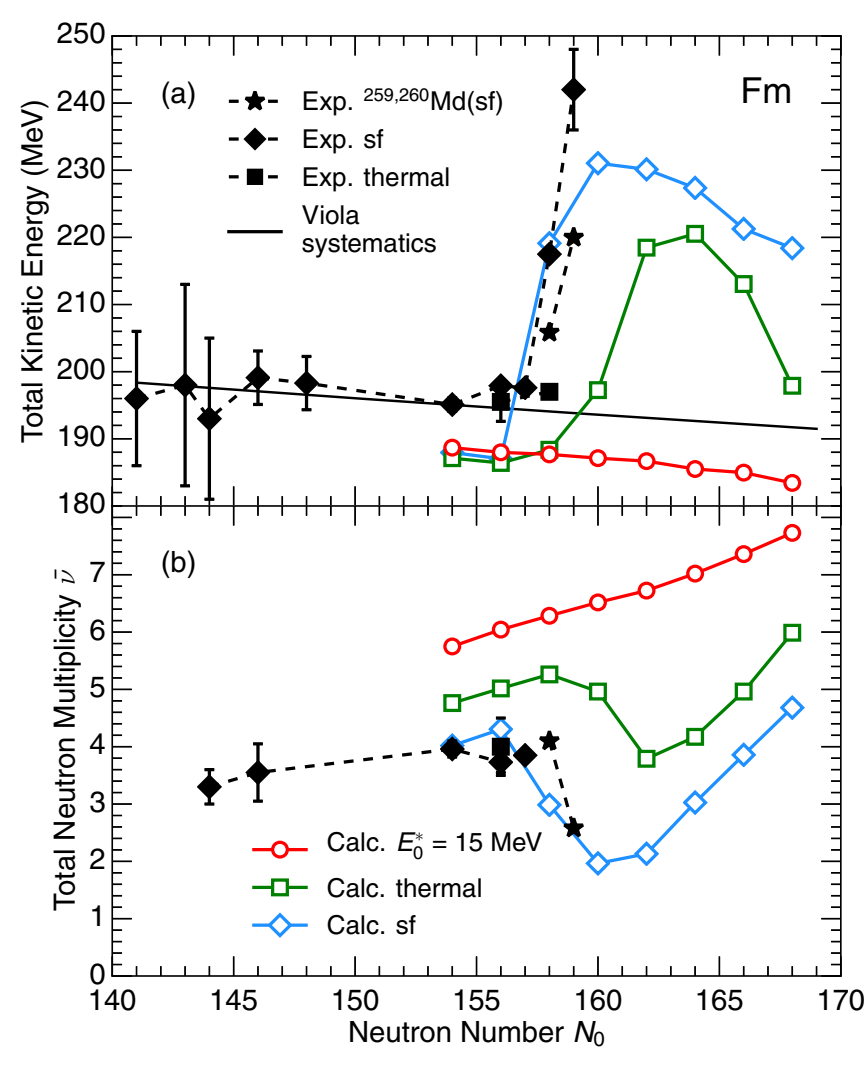

FIG. 6. $\overline{\text { TKE }}$ (a) and $\bar{v}$ (b) for fission of fermium isotopes, vs the neutron number of the initial nucleus. The calculated results for the even isotopes are shown as open blue diamonds for sf, open green squares for thermal fission, and open red circles for $E_{0}^{*}=15 \mathrm{MeV}$. The data on $\overline{\mathrm{TKE}}$ are taken from Refs. [4,7,11,38,43-45] for sf (black diamonds) and from Refs. [5,6] for thermal fission (black squares). The $\bar{v}$ data are taken from Refs. [40,46-48] for sf and from Ref. [41] for thermal fission. Also shown are data for fission of ${ }^{259,260} \mathrm{Md}(\mathrm{sf})$ (black stars), taken from Ref. [11] for $\overline{\mathrm{TKE}}$ and from Refs. [49-51] for $\bar{\nu}$. Data points are connected by dashed lines. The solid line in panel (a) shows the Viola systematics for sf [52].

the energy available for neutron decay [29]. A detailed account of the angular momentum coupling is outside the scope of the present paper.

The average total neutron multiplicity $\bar{v}$ [shown in Fig. 6(b)] mirrors the behavior displayed by TKE [Fig. 6(a)] because, in each fission event, a higher TKE reduces the expected number of neutrons and vice versa. The signature of the super-short fission mode, a low $\bar{v}$, is readily understood from Fig. 2(a) where events with a high TKE appear close to the total available energy $Q_{\mathrm{LH}}^{*}$; this implies low available excitation energies, TXE $=Q_{\mathrm{LH}}^{*}-\mathrm{TKE}$, and thus little energy for the neutrons.

The very low $\bar{v}$ of 2.0 for ${ }^{260} \mathrm{Fm}$ (sf) more than doubles to 5.0 for ${ }^{260} \mathrm{Fm}\left(n_{\text {th }}\right)$ because $f^{\text {ss }}$ is 0.95 for spontaneous fission but only 0.20 for thermal fission. At $E_{0}^{*}=15 \mathrm{MeV}$, fission occurs in the standard mode for all fermium isotopes and $\bar{v}$ increases smoothly from 5.8 for ${ }^{254} \mathrm{Fm}$ to 7.8 for ${ }^{268} \mathrm{Fm}$, in large part due to the decrease of $S_{\mathrm{n}}$ with neutron number.

It has not yet been possible to experimentally test the predicted drop in $\bar{v}$ for sf of fermium isotopes as $N_{0}$ is increased from 156 to 158 , but $\bar{\nu}$ has been measured for ${ }^{259,260} \mathrm{Md}$ (sf) [see Fig. 6(b)] and it decreases from 4.1 to 2.6 as $N_{0}$ goes from 158 to 159.

For fission above the barrier, a more direct comparison can be made between the present results and the results of dynamical calculations solving the Langevin equation [24,25]. For the isotopes of fermium, the general trend is the same as reported here, except that the super-short mode is more pronounced in the Langevin results. Thus, for $E_{0}^{*} \approx 7 \mathrm{MeV}$ fission of ${ }^{258} \mathrm{Fm}$ is predicted to be dominated by the super-short symmetric mode, with less than $5 \%$ asymmetric fission.

\section{SUMMARY}

In summary, we have studied the fission properties of fermium isotopes by simulating the diffusive shape evolution and analyzing the resulting scission configurations. We have focused on the appearance and resilience of the super-short fission mode in which the scission configurations are very compact, consisting of two nearly spherical protofragments near ${ }^{132} \mathrm{Sn}$, thus leading to high fragment kinetic energies and low neutron multiplicities. We have shown in detail how this mode dominates in spontaneous fission of ${ }^{258-268} \mathrm{Fm}$, while it gradually subsides with increasing excitation energy. At energies corresponding to thermal fission only ${ }^{262,264} \mathrm{Fm}$ are still dominated by the super-short mode and for still higher excitation energies it is absent for all isotopes. The calculated results are generally in good agreement with the currently available, limited experimental data.

\section{ACKNOWLEDGMENTS}

Discussions with Karl-Heinz Schmidt are acknowledged. This work was supported by the Knut and Alice Wallenberg Foundation (M.A., B.G.C., and S.Å.). J.R. was supported in part by the NNSA DNN R\&D of the U.S. Department of Energy and its Office of Science under Contract DE-AC02-05CH11231.
[1] M. Bender, R. Bernard, G. Bertsch, S. Chiba, J. Dobaczewski, N. Dubray, S. A. Giuliani, K. Hagino, D. Lacroix, Z. Li et al., J. Phys. G. 47, 113002 (2020).

[2] U. Brosa, S. Grossmann, and A. Müller, Phys. Rep. 197, 167 (1990).

[3] P. Möller, J. R. Nix, and W. J. Swiatecki, Nucl. Phys. A 469, 1 (1987).
[4] J. P. Balagna, G. P. Ford, D. C. Hoffman, and J. D. Knight, Phys. Rev. Lett. 26, 145 (1971).

[5] W. John, E. K. Hulet, R. W. Lougheed, and J. J. Wesolowski, Phys. Rev. Lett. 27, 45 (1971).

[6] R. C. Ragaini, E. K. Hulet, R. W. Lougheed, and J. Wild, Phys. Rev. C 9, 399 (1974). 
[7] E. K. Hulet, R. W. Lougheed, J. H. Landrum, J. F. Wild, D. C. Hoffman, J. Weber, and J. B. Wilhelmy, Phys. Rev. C 21, 966 (1980).

[8] J. F. Wild, E. K. Hulet, R. W. Lougheed, P. A. Baisden, J. H. Landrum, R. J. Dougan, and M. G. Mustafa, Phys. Rev. C 26, 1531 (1982).

[9] H. C. Britt, D. C. Hoffman, J. van der Plicht, J. B. Wilhelmy, E. Cheifetz, R. J. Dupzyk, and R. W. Lougheed, Phys. Rev. C 30, 559 (1984).

[10] E. K. Hulet, J. F. Wild, R. J. Dougan, R. W. Lougheed, J. H. Landrum, A. D. Dougan, M. Schädel, R. L. Hahn, P. A. Baisden, C. M. Henderson, R. J. Dupzyk, K. Sümmerer, and G. R. Bethune, Phys. Rev. Lett. 56, 313 (1986).

[11] E. K. Hulet, J. F. Wild, R. J. Dougan, R. W. Lougheed, J. H. Landrum, A. D. Dougan, P. A. Baisden, C. M. Henderson, R. J. Dupzyk, R. L. Hahn, M. Schädel, K. Sümmerer, and G. R. Bethune, Phys. Rev. C 40, 770 (1989).

[12] S. Cwiok, P. Rozmej, A. Sobiczewski, and Z. Patyk, Nucl. Phys. A 491, 281 (1989).

[13] P. Möller, J. R. Nix, and W. J. Swiatecki, Nucl. Phys. A 492, 349 (1989).

[14] P. Möller and J. R. Nix, J. Phys. G 20, 1681 (1994).

[15] L. Bonneau, Phys. Rev. C 74, 014301 (2006).

[16] N. Dubray, H. Goutte, and J.-P. Delaroche, Phys. Rev. C 77, 014310 (2008).

[17] T. Ichikawa, A. Iwamoto, and P. Möller, Phys. Rev. C 79, 014305 (2009).

[18] J. Zhao, B.-N. Lu, T. Niksic, D. Vretenar, and S. G. Zhou, Phys. Rev. C 93, 044315 (2016).

[19] M. Warda, J. L. Egido, L. M. Robledo, and K. Pomorski, Phys. Rev. C 66, 014310 (2002).

[20] A. Staszczak, A. Baran, J. Dobaczewski, and W. Nazarewicz, Phys. Rev. C 80, 014309 (2009).

[21] D. Regnier, N. Dubray, and N. Schunck, Phys. Rev. C 99, 024611 (2019).

[22] T. Asano, T. Wada, M. Ohta, T. Ichikawa, S. Yamaji, and H. Nakahara, J. Nucl. Radiochem. Sci. 5, 1 (2004).

[23] M. D. Usang, F. A. Ivanyuk, C. Ishizuka, and S. Chiba, Phys. Rev. C 96, 064617 (2017).

[24] Y. Miyamoto, Y. Aritomo, S. Tanaka, K. Hirose, and K. Nishio, Phys. Rev. C 99, 051601(R) (2019).

[25] M. D. Usang, F. A. Ivanyuk, C. Ishizuka, and S. Chiba, Sci. Rep. 9, 1525 (2019).

[26] J. Błocki, Y. Boneh, J. R. Nix, J. Randrup, M. Robel, A. J. Sierk, and W. J. Swiatecki, Ann. Phys. 113, 330 (1978).

[27] J. R. Nix, Nucl. Phys. A 130, 241 (1969).

[28] P. Möller, A. J. Sierk, T. Ichikawa, A. Iwamoto, R. Bengtsson, H. Uhrenholt, and S. Åberg, Phys. Rev. C 79, 064304 (2009).

[29] M. Albertsson, B. G. Carlsson, T. Døssing, P. Möller, J. Randrup, and S. Åberg, Phys. Lett. B 803, 135276 (2020).

[30] M. Albertsson, B. G. Carlsson, T. Døssing, P. Möller, J. Randrup, and S. Åberg, Phys. Rev. C 103, 014609 (2021).

[31] J. Randrup and P. Möller, Phys. Rev. Lett. 106, 132503 (2011).

[32] J. Randrup and P. Möller, Phys. Rev. C 88, 064606 (2013).
[33] J. R. Nix and P. Möller, Nucl. Phys. A 536, 20 (1992)

[34] M. Albertsson, B. G. Carlsson, T. Døssing, P. Möller, J. Randrup, and S. Åberg, Eur. Phys. J. A 56, 46 (2021).

[35] P. Möller and J. Randrup, Phys. Rev. C 91, 044316 (2015).

[36] D. E. Ward, B. G. Carlsson, T. Døssing, P. Möller, J. Randrup, and S. Åberg, Phys. Rev. C 95, 024618 (2017).

[37] A. Bohr and B. R. Mottelson, Nuclear Structure (Benjamin, Reading, MA, 1975), Vol. 2.

[38] J. E. Gindler, K. F. Flynn, L. E. Glendenin, and R. K. Sjoblom, Phys. Rev. C 16, 1483 (1977).

[39] K. F. Flynn, E. P. Horwitz, C. A. A. Bloomquist, R. F. Barnes, R. K. Sjoblom, P. R. Fields, and L. E. Glendenin, Phys. Rev. C 5, 1725 (1972).

[40] D. C. Hoffman, G. P. Ford, J. P. Balagna, and L. R. Veeser, Phys. Rev. C 21, 637 (1980).

[41] K. F. Flynn, J. E. Gindler, R. K. Sjoblom, and L. E. Glendenin, Phys. Rev. C 11, 1676 (1975).

[42] D. C. Hoffman, J. B. Wilhelmy, J. Weber, W. R. Daniels, E. K. Hulet, R. W. Lougheed, J. H. Landrum, J. F. Wild, and R. J. Dupzyk, Phys. Rev. C 21, 972 (1980).

[43] D. C. Hoffman, D. Lee, A. Ghiorso, M. Nurmia, and K. Aleklett, Phys. Rev. C 22, 1581 (1980)

[44] J. P. Unik, J. E. Gindler, L. E. Glendenin, in Proceedings of the Third International Atomic Energy Agency Symposium on the Physics and Chemistry of Fission, Rochester, 2978 (International Atomic Energy Agency, Vienna, 1974), Vol. II, p. 19.

[45] J. Khuyagbaatar, S. Hofmann, F. P. Heßberger, D. Ackermann, H. G. Burkhard, S. Heinz, B. Kindler, I. Kojouharov, B. Lommel, R. Mann, J. Maurer, K. Nishio, and Y. Novikov, Eur. Phys. J. A 37, 177 (2008).

[46] Y. Y. A. Lazarev, At. Energy Rev. 15, 75 (1977).

[47] A. I. Svirikhin, V. N. Dushin, M. L. Chelnokov, V. I. Chepigin, I. N. Izosimov, D. E. Katrasev, O. N. Malyshev, A. Minkova, A. G. Popeko, E. A. Sokol, and A. V. Yeremin, Eur. Phys. J. A 44, 393 (2010).

[48] A. I. Svirikhin, A. V. Andreev, V. N. Dushin, M. L. Chelnokov, V. I. Chepigin, M. Gupta, A. V. Isaev, I. N. Izosimov, D. E. Katrasev, A. N. Kuznetsov, O. N. Malyshev, S. Mullins, A. G. Popeko, E. A. Sokol, and A. V. Yeremin, Eur. Phys. J. A 48, 121 (2012).

[49] A. I. Svirikhin, M. Gupta, A. V. Yeremin, I. N. Izosimov, A. V. Isaev, A. N. Kuznetsov, O. N. Malyshev, S. Mullins, A. G. Popeko, E. A. Sokol, M. L. Chelnokov, and V. I. Chepigin, Bull. Russ. Acad. Sci. Phys. 79, 442 (2015).

[50] J. F. Wild, J. van Aarle, W. Westmeier, R. W. Lougheed, E. K. Hulet, K. J. Moody, R. J. Dougan, E. A. Koop, R. E. Glaser, R. Brandt, and P. Patzelt, Phys. Rev. C 41, 640 (1990).

[51] E. A. Sokol, Cand. Sci. Dissertation, Dubna: JINRI (1992).

[52] V. E. Viola, K. Kwiatkowski, and M. Walker, Phys. Rev. C 31, 1550 (1985).

[53] A. Oberstedt, A. Gatera, A. Göök, and S. Oberstedt, EPJ Web Conf. 211, 04001 (2019).

[54] J. N. Wilson, D. Thisse1, M. Lebois, N. Jovančević, D. Gjestvang, R. Canavan, M. Rudigier, D. Étasse, R.-B. Gerst, L. Gaudefroy et al., Nature (London) 590, 566 (2021). 